\title{
FORCED OSCILLATIONS OF TIMOSHENKO BEAMS
}

BY

TAKAŜI KUSANO ( Hiroshima University)

AND

NORIO YOSHIDA (Iwate University)

1. Introduction. We are concerned with the oscillatory behavior of solutions of the Timoshenko beam equation

$$
\begin{array}{r}
\frac{\partial^{4} u}{\partial t^{4}}+\alpha \beta \gamma \frac{\partial^{2} u}{\partial t^{2}}-(\beta+\gamma) \frac{\partial^{4} u}{\partial x^{2} \partial t^{2}}+\beta \gamma \frac{\partial^{4} u}{\partial x^{4}}+c(x, t, u)=f(x, t), \quad(*) \\
\quad(x, t) \in J \times R_{+},
\end{array}
$$

where $J=(0, L), R_{+}=(0, \infty)$ and $\alpha, \beta, \gamma$ are positive constants. By taking account of the rotary inertia and the deflection due to shear, we obtain the following fourth order differential equation for the transverse vibrations of prismatic beams on elastic foundations:

$$
\frac{\rho^{2} I}{k_{1} G} \frac{\partial^{4} u}{\partial t^{4}}+\rho A \frac{\partial^{2} u}{\partial t^{2}}-\rho\left(I+\frac{E I}{k_{1} G}\right) \frac{\partial^{4} u}{\partial x^{2} \partial t^{2}}+E I \frac{\partial^{4} u}{\partial x^{4}}=q(x, t),
$$

(see [5, p. 433] and [7, p. 150]). If $\alpha=\rho A / E I, \beta=k_{1} G / \rho, \gamma=E / \rho, c(x, t, u) \equiv 0$ and $f(x, t)=\left(k_{1} G / \rho^{2} I\right) q(x, t),(*)$ reduces to Eq. (1).

The purpose of this paper is to obtain sufficient conditions for all solutions of $(*)$ subject to certain boundary conditions to be oscillatory in $J \times R_{+}$. Our method is an adaptation of that used in studying the oscillatory behavior of solutions of hyperbolic equations (cf. [1-3, 6, 8]). In Sec. 2 we present oscillation criteria for a class of fourth order ordinary differential inequalities. In Sec. 3-5 oscillation criteria for $(*)$ are derived on the basis of the results in Sec. 2. We consider three kinds of end conditions, i.e. hinged ends (Sec. 3), hinged-sliding ends (Sec. 4) and sliding ends (Sec. 5).

We assume that the following conditions are satisfied throughout this paper:

(A-I) $c(x, t, \eta)$ is a real-valued continuous function in $J \times R_{+} \times R^{1}$;

(A-II) $\eta c(x, t, \eta) \geqslant 0$ for all $(x, t, \eta) \in J \times R_{+} \times R^{1}$;

(A-III) $c(x, t,-\eta)=-c(x, t, \eta)$ for all $(x, t, \eta) \in J \times R_{+} \times R_{+}$;

(A-IV) $f(x, t)$ is a real-valued continuous function in $J \times R_{+}$.

${ }^{*}$ Received December 5, 1983. 
Definition. A function $u$ : $J \times R_{+} \rightarrow R^{1}$ is said to be oscillatory in $J \times R_{+}$if it has a zero in $J \times(t, \infty)$ for any $t>0$.

2. Fourth order ordinary differential inequalities. We consider the fourth order ordinary differential inequality

$$
y^{(4)}(t)+k^{2} y^{\prime \prime}(t)+m^{2} y(t) \leqslant h(t), \quad t \geqslant a .
$$

The object of this section is to obtain sufficient conditions under which (2) has no eventually positive solution. It is assumed that $k$ and $m$ are nonnegative constants and $h(t)$ is a continuous function on $[a, \infty)$. We define

$$
\omega_{ \pm} \equiv\left(\frac{1}{2}\left(k^{2} \pm\left(k^{4}-4 m^{2}\right)^{1 / 2}\right)\right)^{1 / 2} .
$$

THEOREM 2.1. Inequality (2) has no eventually positive solution if

$$
\liminf _{t \rightarrow \infty} \int_{T}^{t}\left(1-\frac{\xi}{t}\right)^{3} h(\xi) d \xi=-\infty
$$

for all large $T$.

Proof. Suppose to the contrary that there exists a solution $y(t)$ of (2) such that $y(t)>0$ on $\left[t_{0}, \infty\right)$ for some $t_{0}>0$. Integrating $(2)$ over $\left(t_{0}, t\right)$ twice, we obtain

$$
y^{\prime \prime}(t)+k^{2} y(t) \leqslant c_{0}+c_{1} t+\int_{t_{0}}^{t}(t-\xi) h(\xi) d \xi,
$$

where $c_{0}=y^{\prime \prime}\left(t_{0}\right)+k^{2} y\left(t_{0}\right)-\left(y^{(3)}\left(t_{0}\right)+k^{2} y^{\prime}\left(t_{0}\right)\right) t_{0}$ and $c_{1}=k^{2} y^{\prime}\left(t_{0}\right)$. Since

$$
\begin{aligned}
& \int_{t_{0}}^{t}\left(1-\frac{s}{t}\right)\left(\int_{t_{0}}^{s}(s-\xi) h(\xi) d \xi\right) d s \\
&=\int_{t_{0}}^{t} h(\xi)\left(\int_{\xi}^{t}\left(1-\frac{s}{t}\right)(s-\xi) d s\right) d \xi=\int_{t_{0}}^{t} \frac{1}{6 t}(t-\xi)^{3} h(\xi) d \xi,
\end{aligned}
$$

we have

$$
\begin{aligned}
\int_{t_{0}}^{t}\left(1-\frac{s}{t}\right)\left(c_{0}+c_{1} s\right. & \left.+\int_{t_{0}}^{s}(s-\xi) h(\xi) d \xi\right) d s \\
= & t^{2}\left(B\left(t, t_{0}\right)+\frac{1}{6} \int_{t_{0}}^{t}\left(1-\frac{\xi}{t}\right)^{3} h(\xi) d \xi\right),
\end{aligned}
$$

where $B\left(t, t_{0}\right)$ is bounded as $t$ tends to infinity. From (3) and (5) it follows that

$$
\liminf _{t \rightarrow \infty} \int_{t_{0}}^{t}\left(1-\frac{s}{t}\right)\left(c_{0}+c_{1} s+\int_{t_{0}}^{s}(s-\xi) h(\xi) d \xi\right) d s=-\infty .
$$

Applying Theorem 2 of Kusano and Naito [4], we see that under condition (6) inequality (4) has no positive solution on $\left[t_{0}, \infty\right)$. This is a contradiction and the proof is complete. 
Theorem 2.2. Assume that $k^{2}>0, m^{2} \geqslant 0$ and $k^{4}-4 m^{2} \geqslant 0$. Inequality (2) has no eventually positive solution if

$$
\liminf _{t \rightarrow \infty} \int_{T}^{t}\left(1-\frac{s}{t}\right)\left(\int_{s}^{s+\pi / \omega_{+}} h(\xi) \sin \omega_{+}(\xi-s) d \xi\right) d s=-\infty
$$

for all large $T$.

Proof. Suppose that there is a solution $y(t)$ of (2) such that $y(t)>0$ on $\left[t_{0}, \infty\right)$ for some $t_{0}>0$. Letting $\phi=\phi(t, s)=\sin \omega_{+}(t-s)$, we find that $\phi(t, s)>0$ for all $t \in(s, s+$ $\left.\pi / \omega_{+}\right), \phi(s, s)=\phi\left(s+\pi / \omega_{+}, s\right)=0$, and that $\phi(t, s)$ satisfies

$$
\phi_{t t t t}+k^{2} \phi_{t t}+m^{2} \phi=0,
$$

where the subscript $t$ denotes the partial differentiation with respect to $t$. Multiplying (2) by $\phi$ and integrating over $\left(s, s+\pi / \omega_{+}\right)$with respect to $t$, we obtain

$$
\int_{s}^{s+\pi / \omega_{+}} y^{(4)} \phi d t+k^{2} \int_{s}^{s+\pi / \omega_{+}} y^{\prime \prime} \phi d t+m^{2} \int_{s}^{s+\pi / \omega_{+}} y \phi d t \leqslant \int_{s}^{s+\pi / \omega_{+}} h \phi d t .
$$

Integration by parts gives

$$
\begin{aligned}
\int_{s}^{s+\pi / \omega_{+}} y^{\prime \prime} \phi d t= & {\left[y^{\prime} \phi\right]_{s}^{s+\pi / \omega_{+}}-\left[y \phi_{t}\right]_{s}^{s+\pi / \omega_{+}}+\int_{s}^{s+\pi / \omega_{+}} y \phi_{t t} d t } \\
= & \omega_{+}\left(y\left(s+\pi / \omega_{+}\right)+y(s)\right)+\int_{s}^{s+\pi / \omega_{+}} y \phi_{t t} d t, \\
\int_{s}^{s+\pi / \omega_{+}} y^{(4)} \phi d t= & {\left[y^{\prime \prime \prime} \phi\right]_{s}^{s+\pi / \omega_{+}}-\left[y^{\prime \prime} \phi_{t}\right]_{s}^{s+\pi / \omega_{+}}+\left[y^{\prime} \phi_{t t}\right]_{s}^{s+\pi / \omega_{+}} } \\
& -\left[y \phi_{t t t}\right]_{s}^{s+\pi / \omega_{+}}+\int_{s}^{s+\pi / \omega_{+}} y \phi_{t t t t} d t \\
= & \omega_{+}\left(y^{\prime \prime}\left(s+\pi / \omega_{+}\right)+y^{\prime \prime}(s)\right)-\omega_{+}^{3}\left(y\left(s+\pi / \omega_{+}\right)+y(s)\right) \\
& +\int_{s}^{s+\pi / \omega_{+}} y \phi_{t t t t} d t .
\end{aligned}
$$

Combining (9)-(11) and using (8) yields

$$
\begin{aligned}
y^{\prime \prime}\left(s+\pi / \omega_{+}\right)+y^{\prime \prime}(s)+ & \left(k^{2}-\omega_{+}^{2}\right)\left(y\left(s+\pi / \omega_{+}\right)+y(s)\right) \\
& \leqslant \frac{1}{\omega_{+}} \int_{s}^{s+\pi / \omega_{+}} h(\xi) \sin \omega_{+}(\xi-s) d \xi .
\end{aligned}
$$

Since $k^{2}-\omega_{+}^{2}=\omega_{-}^{2} \geqslant 0$, it follows from (12) that $y\left(s+\pi / \omega_{+}\right)+y(s)$ is a positive solution of

$$
z^{\prime \prime}(s)+\omega_{-}^{2} z(s) \leqslant \frac{1}{\omega_{+}} \int_{s}^{s+\pi / \omega_{+}} h(\xi) \sin \omega_{+}(\xi-s) d \xi, \quad s>t_{0} .
$$

On the other hand, using Theorem 2 of [4] and taking account of (7), we conclude that (13) has no eventually positive solution. The contradiction establishes the theorem. 
Theorem 2.3. Assume that $k^{2}>0, m^{2}>0$ and $k^{4}-4 m^{2} \geqslant 0$. Inequality (2) has no eventually positive solution if there exists a nontrivial function $\rho(t) \in C^{2}([1, \infty))$ with the following properties:

(i) $\rho(t)$ is oscillatory on $[1, \infty)$;

(ii) $\rho^{\prime \prime}(t)=\int_{t}^{t+\pi / \omega_{+}} h(\xi) \sin \omega_{+}(\xi-t) d \xi, t \geqslant 1$;

(iii) $\liminf \operatorname{in}_{t \rightarrow \infty} \rho(t)=0$.

Proof. Suppose that (2) has a solution $y(t)$ such that $y(t)>0$ on $\left[t_{0}, \infty\right)$ for some $t_{0}>0$. Proceeding as in the proof of Theorem 2.2, it can be shown that $y\left(s+\pi / \omega_{+}\right)+y(s)$ is a positive solution of $(2)$ on $\left(t_{0}, \infty\right)$. Since $m^{2}>0$, we see that $\omega_{-}>0$. It is known that $z^{\prime \prime}(s)+\omega_{-}^{2} z(s) \leqslant 0$ has no eventually positive solution (see, e.g., Kahane [2, p. 185]). Hence, the hypothesis implies that (13) has no eventually positive solution (see Kusano and Naito [4, Theorem 3]).

TheOREM 2.4. Assume that $k^{2}>0, m^{2}>0$ and $k^{4}-4 m^{2} \geqslant 0$. Inequality (2) has no eventually positive solution if there exists a sequence $\left\{\mathrm{t}_{\mathrm{n}}\right\}$ such that $\lim _{n \rightarrow \infty} t_{n}=\infty$ and

$$
\int_{t_{n}}^{t_{n}+\pi / \omega_{-}}\left(\int_{s}^{s+\pi / \omega_{+}} h(\xi) \sin \omega_{+}(\xi-s) d \xi\right) \sin \omega_{-}\left(s-t_{n}\right) d s \leqslant 0 .
$$

Proof. Let $y(t)$ be a solution of (2) such that $y(t)>0$ on $\left[t_{0}, \infty\right)$ for some $t_{0}>0$. Repeating the argument used in the proof of Theorem 2.2, we observe that $z(s)=y(s+$ $\left.\pi / \omega_{+}\right)+y(s)$ satisfies (13). Multiplying (13) by $\sin \omega_{-}(s-t)$ and integrating over $(t, t+$ $\left.\pi / \omega_{-}\right)$with respect to $s$, we obtain

$$
\begin{array}{r}
y\left(t+\left(\pi / \omega_{+}\right)+\left(\pi / \omega_{-}\right)\right)+y\left(t+\pi / \omega_{+}\right)+y\left(t+\pi / \omega_{-}\right)+y(t) \\
\leqslant\left(\omega_{+} \omega_{-}\right)^{-1} \int_{t}^{t+\pi / \omega_{-}}\left(\int_{s}^{s+\pi / \omega_{+}} h(\xi) \sin \omega_{+}(\xi-s) d \xi\right) \sin \omega_{-}(s-t) d s, \\
t>t_{0},
\end{array}
$$

(cf. Kahane [2, p. 185]). The left-hand side of $(14)$ is positive on $\left(t_{0}, \infty\right)$, and therefore the right-hand side of $(14)$ is also positive on $\left(t_{0}, \infty\right)$. This contradicts the hypothesis and completes the proof.

Remark 1 . In the case where $k^{2}>0, m^{2}>0$ and $k^{4}-4 m^{2} \geqslant 0$, we can replace $\omega_{+}$by $\omega_{-}$in the hypotheses of Theorems 2.2 and 2.3. Theorem 2.4 remains true if $\omega_{-}$is replaced by $\omega_{+}$.

Remark 2. Consider the particular case of (2) in which $h(t) \equiv 0$, i.e.

$$
y^{(4)}(t)+k^{2} y^{\prime \prime}(t)+m^{2} y(t) \leqslant 0 .
$$

If $k^{2}>0, m^{2}>0$ and $k^{4}-4 m^{2} \geqslant 0$, we can apply Theorem 2.4 to conclude that (15) has no eventually positive solution.

3. Hinged ends. We consider the case where the ends of the beam are hinged, so that solutions $u(x, t)$ of $(*)$ are required to satisfy the boundary condition

$$
u(0, t)=\frac{\partial^{2} u}{\partial x^{2}}(0, t)=u(L, t)=\frac{\partial^{2} u}{\partial x^{2}}(L, t)=0, \quad t>0 .
$$


Our main result in this section is the following.

THEOREM 3.1. Every classical solution $u$ of $(*)$ satisfying the boundary condition (HE) is oscillatory in $J \times R_{+}$if the fourth order ordinary differential inequalities

$$
\begin{aligned}
& y^{(4)}(t)+\left(\alpha \beta \gamma+(\beta+\gamma)(\pi / L)^{2}\right) y^{\prime \prime}(t)+\beta \gamma(\pi / L)^{4} y(t) \leqslant F(t), \\
& y^{(4)}(t)+\left(\alpha \beta \gamma+(\beta+\gamma)(\pi / L)^{2}\right) y^{\prime \prime}(t)+\beta \gamma(\pi / L)^{4} y(t) \leqslant-F(t)
\end{aligned}
$$

are oscillatory at $t=\infty$ in the sense that neither (16) nor (17) has a solution which is positive on $[t, \infty)$ for any $t>0$, where

$$
F(t)=\int_{0}^{L} f(x, t) \sin \frac{\pi}{L} x d x .
$$

Proof. Suppose that there is a solution $u$ of the problem (*) and (HE), which has no zero in $J \times\left[t_{0}, \infty\right)$ for some $t_{0}>0$. First, we assume that $u>0$ in $J \times\left[t_{0}, \infty\right)$. By assumption (A-II) we get

$$
\frac{\partial^{4} u}{\partial t^{4}}+\alpha \beta \gamma \frac{\partial^{2} u}{\partial t^{2}}-(\beta+\gamma) \frac{\partial^{4} u}{\partial x^{2} \partial t^{2}}+\beta \gamma \frac{\partial^{4} u}{\partial x^{4}} \leqslant f(x, t) .
$$

Multiplying (18) by $\psi(x)=\sin (\pi / L) x$ and integrating over $(0, L)$, we obtain

$$
\begin{aligned}
& \frac{d^{4}}{d t^{4}} \int_{0}^{L} u \psi(x) d x+\alpha \beta \gamma \frac{d^{2}}{d t^{2}} \int_{0}^{L} u \psi(x) d x \\
& \quad-(\beta+\gamma) \frac{d^{2}}{d t^{2}} \int_{0}^{L} \frac{\partial^{2} u}{\partial x^{2}} \psi(x) d x+\beta \gamma \int_{0}^{L} \frac{\partial^{4} u}{\partial x^{4}} \psi(x) d x \leqslant \int_{0}^{L} f(x, t) \psi(x) d x .
\end{aligned}
$$

Integration by parts and use of (HE) yields

$$
\begin{aligned}
& \int_{0}^{L} \frac{\partial^{2} u}{\partial x^{2}} \psi(x) d x=-(\pi / L)^{2} \int_{0}^{L} u \psi(x) d x, \\
& \int_{0}^{L} \frac{\partial^{4} u}{\partial x^{4}} \psi(x) d x=(\pi / L)^{4} \int_{0}^{L} u \psi(x) d x .
\end{aligned}
$$

Hence, (19) leads to

$$
\begin{aligned}
\frac{d^{4}}{d t^{4}} M[u](t)+\left(\alpha \beta \gamma+(\beta+\gamma)(\pi / L)^{2}\right) \frac{d^{2}}{d t^{2}} M[u](t)+\beta \gamma(\pi / L)^{4} M[u](t) & \\
& \leqslant \int_{0}^{L} f(x, t) \sin \frac{\pi}{L} x d x,
\end{aligned}
$$

where $M[u](t) \equiv \int_{0}^{L} u(x, t) \sin (\pi / L) x d x$. Consequently, we find that $M[u](t)$ is a positive solution of $(16)$ on $\left(t_{0}, \infty\right)$. This contradicts the hypothesis. In the case where $u<0$ on $\left[t_{0}, \infty\right), U \equiv u$ satisfies

$$
\frac{\partial^{4} U}{\partial t^{4}}+\alpha \beta \gamma \frac{\partial^{2} U}{\partial t^{2}}-(\beta+\gamma) \frac{\partial^{4} U}{\partial x^{2} \partial t^{2}}+\beta \gamma \frac{\partial^{4} U}{\partial x^{4}}+c(x, t, U)=-f(x, t) .
$$

Using the same arguments as in the case where $u>0$, we are led to a contradiction. The proof is complete. 
We note that $\alpha \beta \gamma+(\beta+\gamma)(\pi / L)^{2}>0, \beta \gamma(\pi / L)^{4}>0$ and

$$
\begin{aligned}
\left(\alpha \beta \gamma+(\beta+\gamma)(\pi / L)^{2}\right)^{2} & -4 \beta \gamma(\pi / L)^{4} \\
& =(\alpha \beta \gamma)^{2}+2 \alpha \beta \gamma(\beta+\gamma)(\pi / L)^{2}+(\beta-\gamma)^{2}(\pi / L)^{4}>0 .
\end{aligned}
$$

We define the constants $\tilde{\omega}_{ \pm}$by

$$
\begin{aligned}
\tilde{\omega}_{ \pm} \equiv & 2^{-1 / 2}\left(\alpha \beta \gamma+(\beta+\gamma)(\pi / L)^{2}\right. \\
& \left. \pm\left((\alpha \beta \gamma)^{2}+2 \alpha \beta \gamma(\beta+\gamma)(\pi / L)^{2}+(\beta-\gamma)^{2}(\pi / L)^{4}\right)^{1 / 2}\right)^{1 / 2} .
\end{aligned}
$$

Applying Theorems $2.1-2.4$ to (16) and (17), we obtain the following results.

COROLlaRy 3.1. Every classical solution $u$ of $(*)$ satisfying (HE) is oscillatory in $J \times R_{+}$if

$$
\begin{gathered}
\liminf _{t \rightarrow \infty} \int_{T}^{t}\left(1-\frac{\xi}{t}\right)^{3} F(\xi) d \xi=-\infty \\
\limsup _{t \rightarrow \infty} \int_{T}^{t}\left(1-\frac{\xi}{t}\right)^{3} F(\xi) d \xi=\infty
\end{gathered}
$$

for all large $T$.

COROllary 3.2. Every classical solution $u$ of $(*)$ satisfying (HE) is oscillatory in $J \times R_{+}$if

$$
\begin{aligned}
\liminf _{t \rightarrow \infty} \int_{T}^{t}\left(1-\frac{s}{t}\right)\left(\int_{s}^{s+\pi / \tilde{\omega}} F(\xi) \sin \tilde{\omega}(\xi-s) d \xi\right) d s & =-\infty, \\
\limsup _{t \rightarrow \infty} \int_{T}^{t}\left(1-\frac{s}{t}\right)\left(\int_{s}^{s+\pi / \omega_{+}} F(\xi) \sin \tilde{\omega}(\xi-s) d \xi\right) d s & =\infty
\end{aligned}
$$

for all large $T$, where $\tilde{\omega}=\tilde{\omega}_{+}$or $\tilde{\omega}=\tilde{\omega}_{-}$.

COROllary 3.3. Every classical solution $u$ of $(*)$ satisfying (HE) is oscillatory in $J \times R_{+}$if there exists a nontrivial function $\rho(t) \in C^{2}([1, \infty))$ with the following properties:

(i) $\rho(t)$ is oscillatory on $[1, \infty)$;

(ii) $\rho^{\prime \prime}(t)=\int_{t}^{t+\pi / \bar{\omega}} F(\xi) \sin \tilde{\omega}(\xi-t) d \xi, t \geqslant 1$;

(iii) $\lim _{t \rightarrow \infty} \rho(t)=0$,

where $\tilde{\omega}=\tilde{\omega}_{+}$or $\tilde{\omega}=\tilde{\omega}_{-}$.

COROLlary 3.4. Every classical solution $u$ of $(*)$ satisfying (HE) is oscillatory in $J \times R_{+}$if

$$
\int_{t}^{t+\pi / \tilde{\omega}_{-}}\left(\int_{s}^{s+\pi / \tilde{\omega}_{+}} F(\xi) \sin \tilde{\omega}_{+}(\xi-s) d \xi\right) \sin \tilde{\omega}_{-}(s-t) d s
$$

is oscillatory on $\left(t_{0}, \infty\right)$ for some $t_{0}>0$.

Example 1. We consider the equation

$$
\frac{\partial^{4} u}{\partial t^{4}}+\frac{4}{3} \frac{\partial^{2} u}{\partial t^{2}}-\frac{2}{3}(L / \pi)^{2} \frac{\partial^{4} u}{\partial x^{2} \partial t^{2}}+\frac{1}{9}(L / \pi)^{4} \frac{\partial^{4} u}{\partial x^{4}}=-\frac{8}{9}\left(\sin \frac{\pi}{L} x\right) t^{4} \sin t
$$


Here $\alpha=12(\pi / L)^{4}, \beta=\gamma=\frac{1}{3}(L / \pi)^{2}$ and $c(x, t, u) \equiv 0$. A direct calculation shows that

$$
\begin{aligned}
\int_{T}^{t}\left(1-\frac{\xi}{t}\right)^{3}\left(\int_{0}^{L}-\frac{8}{9} \xi^{4}(\sin \xi)\right. & \left.\left(\sin \frac{\pi}{L} x\right)^{2} d x\right) d \xi \\
& =-\frac{4}{9} L \int_{T}^{t}\left(1-\frac{\xi}{t}\right)^{3} \xi^{4} \sin \xi d \xi=-\frac{8}{3} L t \sin t+B(t, T),
\end{aligned}
$$

where $B(t, T)$ is bounded as $t$ tends to infinity. Hence, we obtain

$$
\begin{aligned}
& \liminf _{t \rightarrow \infty} \int_{T}^{t}\left(1-\frac{\xi}{t}\right)^{3}\left(-\frac{8}{9} \int_{0}^{L} \xi^{4}(\sin \xi)\left(\sin \frac{\pi}{L} x\right)^{2} d x\right) d \xi=-\infty, \\
& \limsup _{t \rightarrow \infty} \int_{T}^{t}\left(1-\frac{\xi}{t}\right)^{3}\left(-\frac{8}{9} \int_{0}^{L} \xi^{4}(\sin \xi)\left(\sin \frac{\pi}{L} x\right)^{2} d x\right) d \xi=\infty
\end{aligned}
$$

It follows from Corollary 3.1 that every classical solution of (20) satisfying (HE) is oscillatory in $J \times R_{+}$. In fact, there exists an oscillatory solution of (20)

$$
u=\left(\sin \frac{\pi}{L} x\right)\left(t^{4} \sin t-54 t^{2} \sin t+108 t \cos t+513 \sin t\right)
$$

which satisfies (HE).

Example 2. We consider the equation

$$
\frac{\partial^{4} u}{\partial t^{4}}+4 \frac{\partial^{2} u}{\partial t^{2}}-4(L / \pi)^{2} \frac{\partial^{4} u}{\partial x^{2} \partial t^{2}}+4(L / \pi)^{4} \frac{\partial^{4} u}{\partial x^{4}}=16\left(\sin \frac{\pi}{L} x\right) e^{-t} \sin t
$$

Here $\alpha=(\pi / L)^{4}, \beta=\gamma=2(L / \pi)^{2}$ and $c(x, t, u) \equiv 0$. Since

$$
\begin{aligned}
& \left|\int_{T}^{t}\left(1-\frac{\xi}{t}\right)^{3}\left(\int_{0}^{L} 16 e^{-\xi}(\sin \xi)\left(\sin \frac{\pi}{L} x\right)^{2} d x\right) d \xi\right| \\
& \leqslant 8 L \int_{T}^{t}\left|\left(1-\frac{\xi}{t}\right)^{3} e^{-\xi} \sin \xi\right| d \xi \leqslant 8 L \int_{0}^{\infty} e^{-\xi} d \xi=8 L
\end{aligned}
$$

Corollary 3.1 is not applicable to (21). However, we can apply Corollary 3.3 to (21). Integration by parts gives

$$
\begin{aligned}
\int_{t}^{t+\pi / \tilde{\omega}} F(\xi) \sin \tilde{\omega}(\xi-t) d \xi=8 L \int_{t}^{t+\pi / \tilde{\omega}} e^{-\xi}(\sin \xi) \sin \tilde{\omega}(\xi-t) d \xi \\
=A e^{-t} \cos (t+\pi / \tilde{\omega})+B e^{-t} \sin (t+\pi / \tilde{\omega})+C e^{-t} \cos t+D e^{-t} \sin t,
\end{aligned}
$$

where $A=16 L \tilde{\omega}\left(\tilde{\omega}^{4}+4\right)^{-1} e^{-\pi / \tilde{\omega}}, B=8 L \tilde{\omega}^{3}\left(\tilde{\omega}^{4}+4\right)^{-1} e^{-\pi / \tilde{\omega}}, C=16 L \tilde{\omega}\left(\tilde{\omega}^{4}+4\right)^{-1}$ and $D=8 L \tilde{\omega}^{3}\left(\tilde{\omega}^{4}+1\right)^{-1}$. Defining

$$
\rho(t) \equiv \frac{1}{2} e^{-t}(-A \sin (t+\pi / \tilde{\omega})+B \cos (t+\pi / \tilde{\omega})-C \sin t+D \cos t),
$$

we see that $\rho(t)$ satisfies conditions (i)-(iii) of Corollary 3.3. Hence, every classical solution $u$ of (21) satisfying (HE) is oscillatory in $J \times R_{+}$. In fact, Eq. (21) has an oscillatory solution $u=(\sin (\pi / L) x) e^{-t} \cos t$ which satisfies (HE).

Example 3. Consider the equation

$$
\frac{\partial^{4} u}{\partial t^{4}}+2(\pi / L)^{4} \frac{\partial^{2} u}{\partial t^{2}}-2(\pi / L)^{2} \frac{\partial^{4} u}{\partial x^{2} \partial t^{2}}+(\pi / L)^{4} \frac{\partial^{4} u}{\partial x^{4}}=0 .
$$


Here $\alpha=2, \beta=\gamma=(\pi / L)^{2}$ and $c(x, t, u)=f(x, t) \equiv 0$. Since $f(x, t) \equiv 0$, we have $F(t) \equiv 0$. Therefore, Corollaries 3.1-3.3 are not applicable to (22). However, Corollary 3.4 implies that every classical solution $u$ of (22) satisfying (HE) is oscillatory in $J \times R_{+}$. In fact, there exists an oscillatory solution $u=(\sin (\pi / L) x) \cos \left(\sqrt{2+\sqrt{3}}(\pi / L)^{2} t\right)$ which satisfies (HE).

4. Hinged-sliding ends. In this section we deal with the case of hinged-sliding ends for which the boundary condition takes the form

$$
u(0, t)=\frac{\partial^{2} u}{\partial x^{2}}(0, t)=\frac{\partial u}{\partial x}(L, t)=\frac{\partial^{3} u}{\partial x^{3}}(L, t)=0, \quad t>0 .
$$

We obtain the following analogue of Theorem 3.1.

THEOREM 4.1. Every classical solution $u$ of (*) satisfying the boundary condition (HSE) is oscillatory in $J \times R_{+}$if the fourth order ordinary differential inequalities

$$
\begin{aligned}
& y^{(4)}(t)+\left(\alpha \beta \gamma+(\beta+\gamma)(\pi /(2 L))^{2}\right) y^{\prime \prime}(t)+\beta \gamma(\pi /(2 L))^{4} y(t) \leqslant G(t), \\
& y^{(4)}(t)+\left(\alpha \beta \gamma+(\beta+\gamma)(\pi /(2 L))^{2}\right) y^{\prime \prime}(t)+\beta \gamma(\pi /(2 L))^{4} y(t) \leqslant-G(t)
\end{aligned}
$$

are oscillatory at $t=\infty$ in the sense that neither (23) nor (24) has a solution which is positive on $[t, \infty)$ for any $t>0$, where

$$
G(t)=\int_{0}^{L} f(x, t) \sin \frac{\pi}{2 L} x d x
$$

Proof. Suppose that the problem (*) and (HSE) has a solution $u$ which has no zero in $J \times\left[t_{0}, \infty\right)$ for some $t_{0}>0$. We may suppose that $u>0$ in $J \times\left[t_{0}, \infty\right)$. As in the proof of Theorem 3.1, we obtain the inequality (18). Multiplying (18) by $\tilde{\psi}(x)=\sin (\pi /(2 L)) x$ and integrating over $(0, L)$, we have (19) with $\psi(x)$ replaced by $\tilde{\psi}(x)$. Integration by parts gives

$$
\begin{aligned}
& \int_{0}^{L} \frac{\partial^{2} u}{\partial x^{2}} \tilde{\psi}(x) d x=-(\pi /(2 L))^{2} \int_{0}^{L} u \tilde{\psi}(x) d x, \\
& \int_{0}^{L} \frac{\partial^{4} u}{\partial x^{4}} \tilde{\psi}(x) d x=(\pi /(2 L))^{4} \int_{0}^{L} u \tilde{\psi}(x) d x .
\end{aligned}
$$

Hence, $\int_{0}^{L} u \tilde{\psi}(x) d x$ is a positive solution of $(23)$ on $\left(t_{0}, \infty\right)$. This contradicts the hypothesis.

By specializing the results in Sec. 2 to (23) and (24), one could easily formulate various oscillation criteria for equation (*) plus (HSE).

5. Sliding ends. We consider the case of sliding ends, i.e. equation (*) subject to the boundary condition

$$
\frac{\partial u}{\partial x}(0, t)=\frac{\partial^{3} u}{\partial x^{3}}(0, t)=\frac{\partial u}{\partial x}(L, t)=\frac{\partial^{3} u}{\partial x^{3}}(L, t)=0, \quad t>0 .
$$


THEOREM 5.1. Every classical solution $u$ of $(*)$ satisfying the boundary condition (SE) is oscillatory in $J \times R_{+}$if the fourth order ordinary differential inequalities

$$
\begin{aligned}
& y^{(4)}(t)+\alpha \beta \gamma y^{\prime \prime}(t) \leqslant H(t), \\
& y^{(4)}(t)+\alpha \beta \gamma y^{\prime \prime}(t) \leqslant-H(t)
\end{aligned}
$$

are oscillatory at $t=\infty$, where

$$
H(t)=\int_{0}^{L} f(x, t) d x .
$$

Proof. Let $u$ be a solution of the problem (*) and (SE), which has no zero in $J \times\left[t_{0}, \infty\right)$ for some $t_{0}>0$. First we assume $u>0$ in $J \times\left[t_{0}, \infty\right)$. As in the proof of Theorem 3.1, we obtain the inequality (18). Integrating $(18)$ over $(0, L)$, we have

$$
\begin{aligned}
\frac{d^{4}}{d t^{4}} \int_{0}^{L} u d x+\alpha \beta \gamma \frac{d^{2}}{d t^{2}} \int_{0}^{L} u d x-(\beta+\gamma) & \frac{d^{2}}{d t^{2}} \int_{0}^{L} \frac{\partial^{2} u}{\partial x^{2}} d x \\
& +\beta \gamma \int_{0}^{L} \frac{\partial^{4} u}{\partial x^{4}} d x \leqslant \int_{0}^{L} f(x, t) d x
\end{aligned}
$$

Since

$$
\begin{aligned}
& \int_{0}^{L} \frac{\partial^{2} u}{\partial x^{2}} d x=\frac{\partial u}{\partial x}(L, t)-\frac{\partial u}{\partial x}(0, t)=0 \\
& \int_{0}^{L} \frac{\partial^{4} u}{\partial x^{4}} d x=\frac{\partial^{3} u}{\partial x^{3}}(L, t)-\frac{\partial^{3} u}{\partial x^{3}}(0, t)=0,
\end{aligned}
$$

(27) reduces to

$$
\frac{d^{4}}{d t^{4}} \int_{0}^{L} u d x+\alpha \beta \gamma \frac{d^{2}}{d t^{2}} \int_{0}^{L} u d x \leqslant \int_{0}^{L} f(x, t) d x .
$$

This shows that (25) has a positive solution $\int_{0}^{L} u d x$ on $\left(t_{0}, \infty\right)$, contradicting the hypothesis. In the case where $u<0$ in $J \times\left[t_{0}, \infty\right), U \equiv-u$ satisfies

$$
\frac{\partial^{4} U}{\partial t^{4}}+\alpha \beta \gamma \frac{\partial^{2} U}{\partial t^{2}}-(\beta+\gamma) \frac{\partial^{4} U}{\partial x^{2} \partial t^{2}}+\beta \gamma \frac{\partial^{4} U}{\partial x^{4}}+c(x, t, U)=-f(x, t) .
$$

The same argument as above leads us to a contradiction. The proof is complete.

ThEOREM 5.2. Assume that $c(x, t, u)=p_{0} u$ ( $p_{0}$ is a positive constant), i.e.

$$
\frac{\partial^{4} u}{\partial t^{4}}+\alpha \beta \gamma \frac{\partial^{2} u}{\partial t^{2}}-(\beta+\gamma) \frac{\partial^{4} u}{\partial x^{2} \partial t^{2}}+\beta \gamma \frac{\partial^{4} u}{\partial x^{4}}+p_{0} u=f(x, t) .
$$

Every classical solution $u$ of (29) satisfying (SE) is oscillatory in $J \times R_{+}$if $0<p_{0}$ $\leqslant \frac{1}{4}(\alpha \beta \gamma)^{2}$ and the function

$$
\int_{t}^{t+\pi / \omega_{-}}\left(\int_{s}^{s+\pi / \omega_{+}} H(\xi) \sin \omega_{+}(\xi-s) d \xi\right) \sin \omega_{-}(s-t) d s
$$


is oscillatory on $\left(t_{0}, \infty\right)$ for some $t_{0}>0$, where

$$
\omega_{ \pm}=\left(\frac{1}{2}\left(\alpha \beta \gamma \pm\left((\alpha \beta \gamma)^{2}-4 p_{0}\right)^{1 / 2}\right)\right)^{1 / 2} .
$$

Proof. In the case where $u>0$ in $J \times\left[t_{0}, \infty\right)$ for some $t_{0}>0$, we obtain

$$
\frac{d^{4}}{d t^{4}} \int_{0}^{L} u d x+\alpha \beta \gamma \frac{d^{2}}{d t^{2}} \int_{0}^{L} u d x+p_{0} \int_{0}^{L} u d x \leqslant H(t)
$$

instead of (28). Proceeding as in the proof of Theorem 5.1, we find that the conclusion follows from Theorem 2.4 .

Corollary 5.1. Every classical solution $u$ of $(*)$ satisfying (SE) is oscillatory in $J \times R_{+}$if

$$
\begin{aligned}
& \liminf _{t \rightarrow \infty} \int_{T}^{t}\left(1-\frac{\xi}{t}\right)^{3} H(\xi) d \xi=-\infty, \\
& \limsup _{t \rightarrow \infty} \int_{T}^{t}\left(1-\frac{\xi}{t}\right)^{3} H(\xi) d \xi=\infty
\end{aligned}
$$

for all large $T$.

Proof. By Theorem 2.1 we see that (25) and (26) are oscillatory at $t=\infty$. The conclusion follows from Theorem 5.1.

Corollary 5.2. Every classical solution $u$ of $(*)$ satisfying (SE) is oscillatory in $J \times R_{+}$if

$$
\begin{gathered}
\liminf _{t \rightarrow \infty} \int_{T}^{t}\left(1-\frac{s}{t}\right)\left(\int_{s}^{s+\pi / \omega} H(\xi) \sin \omega(\xi-s) d \xi\right) d s=-\infty, \\
\limsup _{t \rightarrow \infty} \int_{T}^{t}\left(1-\frac{s}{t}\right)\left(\int_{s}^{s+\pi / \omega} H(\xi) \sin \omega(\xi-s) d \xi\right) d s=\infty
\end{gathered}
$$

for all large $T$, where $\omega=(\alpha \beta \gamma)^{1 / 2}$.

Proof. We observe, using Theorem 2.2, that (25) and (26) are oscillatory at $t=\infty$. The conclusion follows from Theorem 5.1.

Remark 3. We consider the equation (29) with $f(x, t) \equiv 0$, i.e.

$$
\frac{\partial^{4} u}{\partial t^{4}}+\alpha \beta \gamma \frac{\partial^{2} u}{\partial t^{2}}-(\beta+\gamma) \frac{\partial^{4} u}{\partial x^{2} \partial t^{2}}+\beta \gamma \frac{\partial^{4} u}{\partial x^{4}}+p_{0} u=0
$$

It follows from Theorem 5.2 that every classical solution $u$ of (30) satisfying (SE) is oscillatory in $J \times R_{+}$if $0<p_{0} \leqslant \frac{1}{4}(\alpha \beta \gamma)^{2}$.

\section{REFERENCES}

[1] C. Y. Chan and E. C. Young. Comparison theorems for a coupled system of singular hyperholic differential inequalities. I. Time-independent uncoupling coefficients, Atti. Accad. Naz. Lincei Rend. Cl. Sci. Fis. Mat. Natur. 66, 250-254 (1979)

[2] C. Kahane, Oscillation theorems for solutions of hyperbolic equations, Proc. Amer. Math. Soc. 41, 183-188 (1973)

[3] K. Kreith, Oscillation theory, Lecture Notes in Mathematics, vol. 324, Springer-Verlag, Berlin, 1973 
[4] T. Kusano and M. Naito, Oscillation criteria for a class of perturbed Schrödinger equations, Canad. Math. Bull. 25, 71-77 (1982)

[5] S. Timoshenko, D. H. Young and W. Weaver, Jr., Vibration problems in engineering, Fourth Edition, Wiley, New York, 1974

[6] C. C. Travis, Comparison and oscillation theorems for hyperbolic equations, Utilitas Math. 6, 139-151 (1974)

[7] T. M. Wang and J. E. Stephens, Natural frequencies of Timoshenko beams on Pasternak foundations, J. Sound and Vibration 51, 149-155 (1977)

[8] E. C. Young, Comparison and oscillation theorems for singular hyperbolic equations, Atti Accad. Naz. Lincei Rend. Cl. Sci. Fis. Mat. Natur. 59, 383-391 (1975) 